\title{
Clinical evaluation of zinc-carbonate hydroxyapatite nanocrystals mouthwash in controlling plaque induced gingivitis: A randomized clinical trial
}

\author{
Dhafer Al Asmari ${ }^{*}$, Abdullah Almutairi ${ }^{2}$ \\ Assistant Professor, Dept. of Periodontology \& Oral Medicine, College of Dentistry, Qassim University, Saudi Arabia \\ *Corresponding Author: Dhafer Al Asmari \\ Email: dr.dhafer.alasmari@qudent.org
}

\begin{abstract}
Objectives: We evaluated the efficacy of Zinc-carbonate hydroxyapatite nanocrystals $(\mathrm{ZCH})$ mouthwash (Biorepair ${ }^{\circledR}$ Mouthwash) in controlling plaque and gingivitis comparing with chlorhexidine mouthwashes.

Design: Single center double blind parallel randomized clinical trial.

Setting: Qassim University, College of Dentistry clinics.

Participants: 88 patients with plaque induced gingivitis. 52 patients fulfilled the study requirements.

Intervention: Use of mouthwash twice a day for 60s for 2 weeks. Test group used Zinc-carbonate hydroxyapatite nanocrystals (ZCH) mouthwash (Biorepair ${ }^{\circledR}$ Mouthwash) while control group used chlorhexidine mouthwashes. Follow-up 2 weeks.

Main outcome measures: Gingival index and Plaque index.

Results: 52 patients (control $n=26$; test $n=26$ ) were randomized. One subject in each group were lost to follow up, leaving 50 patients (control $n=25$; test $n=25$ ) for analysis. Mean Gingival and Plaque Index score at 2 nd week after the use of test and control mouthwash was statically not significant, $\mathrm{p}<0.006$ and $\mathrm{p}<0.503$ respectively however, there is marked reduction percentage wise in the Gingival and Plaque Index score in both groups.

Conclusions: Zinc-carbonate hydroxyapatite nanocrystals $(\mathrm{ZCH})$ and Chlorhexidine Mouthwash $(\mathrm{CHX})$ both were effective in controlling Dental Plaque and Gingivitis.
\end{abstract}

Keywords: Zn-carbonate hydroxyapatite nanocrystals, Mouthwash, Chlorhexidine, Plaque, Gingivitis.

\section{Introduction}

Now days oral hygiene products play an important role in dental practice for maintaining good oral health. For prescribing these products requires scientifically documented clinical efficacy of the products. Dentifrices and mouth-rinse need more study in terms of their antiplaque and anti-gingivitis properties.

Mouthwashes are extensively used as an adjunct means for maintaining oral hygiene. These mouthwashes play an important role in controlling dental plaque by inhibiting growth of microorganisms and blocking enzymatic activity (Saad, Greenman, \& Shaw, 2011).

It is principally agreed that dental plaque biofilm is the key etiologic factor initiating "Gingivitis" and justify the use of antimicrobial mouthwashes (Löe, Theilade, \& Jensen, 1965) Willoughby D. Miller (1880) was the first "Dentist" to propose the use of phenolic compounds in mouthwash to control "Gingivitis".7 (Jackson, 1997).

Patients are comfortable/easy to use these mouthwashes, because of their properties of controlling dental plaque biofilm and fresh breath effects (Diane Cummins, 1997; D Cummins \& Creeth, 1992; Moran, 1997). Dental biofilm is the main cause for dental Caries, Gingivitis, and Periodontitis" (Kuboniwa \& Lamont, 2010; Noiri, Li, \& Ebisu, 2001; Page, Offenbacher, Schroeder, Seymour, \& Kornman, 1997; Pitts et al., 2017; Takahashi \& Nyvad, 2011).

Dental biofilm can be control by mechanical means like tooth brushing and flossing (Socransky \& Haffajee, 2002). In order to provide extra effects for these mechanical tools of controlling plaque, antimicrobial substances are used in
"Oral Care Products" as mouthwash or dentifrices (Brading \& Marsh, 2003; Marsh, 2010, 2012). In reviews, it is to be proved that mouthwash have role in improving gingival inflammation and plaque control without effect on any microbial resistance and changes to "Microbial Flora" (Sreenivasan \& Gaffar, 2002). Dental Biofilm contains pathogens responsible periodontal disease and dental caries are for most widespread microbial, therefore plaque control very important for oral health (Seymour, Ford, Cullinan, Leishman, \& Yamazaki, 2007).

Organization of the dental plaque film with the help of tooth brushing/flossing is not the way to obtain objectively, some adjuncts are required to for remineralization (M. Hannig \& Hannig, 2010; Hassanein \& El-Brolossy, 2006; Marsh, 2004). Hydroxyapatite nanocrystals were industrialized due to current revolution in nanotechnology, and responsible to form a layer, which protects enamel structure (Roveri et al., 2008).

Zinc-carbonate hydroxyapatite nanocrystals $(\mathrm{ZCH})$ mouthwash (Biorepair $®$ Mouthwash, Coswell, Italy) is a products claim with a successful market history on the basis of multipurpose uses. However, there are few publications on the clinical efficacy of this product is available. An online search of the relevant scientific literature (PubMed, up to April 2017) found only one article on clinical efficacy of Zinc-carbonate hydroxyapatite nanocrystals ( $\mathrm{ZCH})$ mouthwash (Biorepair® Mouthwash)(C. Hannig, Basche, Burghardt, Al-Ahmad, \& Hannig, 2013). This study was published in 2013 and concerned new preparation in dental prophylaxis containing zinc-carbonate hydroxyapatite microclusters (Biorepair) for oral biofilm management. One 
study found on Google Scholar in which researcher compare the effectiveness of Biorepair, fluoride and chlorhexidine mouthwashes in controlling plaque accumulation and gingivitis (Hegazy \& Salama, 2016).

Biorepair mouthwash contains "Aqua, Sorbitol, Glycerin, Xylitol, Cellulose Gum, Zinc PCA, Zinc Hydroxyapatite, Aroma, Sodium Lauryl Sulfate, Silica, Ricinus Communis Seed Oli, Ammonium Acryloyldimethyltaurate/VP Copolymer, Mentha Arvensis Leaf Oil, Sodium Myristoyl Sarcosinate, Sodium Methyl Cocoyl Taurate, Sodium Saccharin, Tromethamine, Sodium Benzoate, Benzyl Alcohol, Phenoxyethanol, Limonene. microRepair". Zinc Hydroxyapatite is an effective compound for biofilm management in the oral cavity due to antiadherent and antibacterial effects (C. Hannig et al., 2013).

Due to limited research studies on this product in vivo, suggest the need for clinical documentation of the antiplaque and anti-gingivitis efficacy of Zinc-carbonate hydroxyapatite nanocrystals $(\mathrm{ZCH})$ mouthwash. The purpose of the proposed clinical trial is to rapidly screen for the efficacy of Zinc-carbonate hydroxyapatite nanocrystals (ZCH) mouthwash (Biorepair® Mouthwash) in controlling plaque and gingivitis comparing with chlorhexidine mouthwashes.

\section{Materials and Methods}

This study was a 2-week single center; double blind randomize control clinical trial. After complete screening patients of "Plaque Induced Gingivitis" fulfill all inclusion criteria were included in the study. The Ethical Committee at the College of Dentistry Qassim University approved this study.

Sample size calculations were made for a single group (paired t-test), using the following assumptions: mean ( \pm SD) baseline GI level of $1.2( \pm 0.35)$ and a post-treatment GI of $0.91( \pm 0.40)$, considered clinically relevant, with a level of significance of $\alpha=0.05$ and a power of $80 \%$, at least 40 subjects need to be included in the study. Assuming a dropout rate of up to $25 \%$, the total need to enroll 52 subjects for study. At the end of two subject failed to complete the study, data will analysis on 50 subjects (control $n=25$, test $n=25$ ) who complete the study as per protocol.

\section{Inclusion criteria}

Male and female subjects were 30 to 60 years of age in good health, and must have 20 teeth were included (Table 1). Full mouth Gingival Index (GI) score $\geq 1.0$ and willing to sign approved informed consent.

\section{Exclusion criteria}

Third molars, grossly carious, fully crowned/restored, and abutment teeth are excluded from count. Current user of Biorepair mouthwash or any other mouthrinse not include in the study. Patients with any removable appliance (e.g., removable partial denture, orthodontic appliance), fixed orthodontic appliances (including permanent orthodontic retainers) and Probing Depth (PD) > $4 \mathrm{~mm}$ at any site were also not include in the study. Patients should not be included if allergy to any ingredients of the products, use of antibiotic in the last 3 months, anticoagulant medication (Heparin, aspirin), anti-inflammatory medication (aspirin, ibuprofen, naproxen), and medications known to have effects on the gingiva (phenytoin, calcium channel blockers, cyclosporine)

\section{Study design}

The study will consist of the following appointments for clinical examinations:

1. Baseline examination: 1- 2 weeks following screening appointment.

2. Final examination: 2 weeks following baseline appointment subject to use of experimental material as per instructions.

Two trained and calibrated examiners (Kappa score 0.88 ) performed all clinical examinations and record clinical parameters at dental clinics in College of Dentistry, Qassim university. The clinical oral examination assessed whether the subject has fulfilled the inclusion criteria.

At baseline, clinical oral examination of the all subjects of the study was performed and allocate study materials (New Toothbrush, Test or Control product) with study instructions. Study material was kept in a sealed envelope with coding, for distributing to the participating subjects randomly. After baseline examination, examiner's hand over the data form to the material distributing person, with instruction to write the code of envelope on the recording form.

At baseline record the Gingival Inflammation, using the Gingival Index (GI) Löe and Silness (Loe \& Silness, 1963) modified by Talbott et al (Talbott, Mandel, \& Chilton, 1977). Also record Plaque index (PI), using the Turesky modification (Turesky, Gilmore, \& Glickman, 1970) of the Quigley-Hein Index (Quigley \& Hein, 1962). Subject included in the study provides new toothbrush and one of the two study products (Test or control) randomly. All participants were asked to abstain from all mechanical plaque-control measures but to rinse twice a day with $10 \mathrm{ml}$ of the assigned solution (test: $\mathrm{ZCH}, \quad$ (Biorepair ${ }^{\circledR}$ Mouthwash, Coswell, Italy) mouthwash, control: $0.1 \%$ chlorhexidine (CHX)) for $60 \mathrm{~s}$.

After 2 weeks following the baseline examination subject to use of experimental material as per instructions, study subjects will present for the final clinical oral examination. In this examination, record the same clinical parameters GI and PI by a blinded examiner.

\section{Statistical analysis}

Data were calculated statistical software SPSS version 22, results were presented for each of the following outcomes: "Gingivitis, and Dental Plaque". As the data was distributed, normal, Independent t-test was applied on comparison of mean in both groups for each variable.

\section{Results}

The mean age of patients in control and test group was $41.04 \pm 6.91$ years and $39.52 \pm 5.58$ years with no significant difference, p-value $>0.05$ (Table 1). In control group, there were $16(64 \%)$ male and $9(36 \%)$ female cases 
while test group there were $17(68 \%)$ male and $8(32 \%)$ female cases with significant difference, $\mathrm{p}$-value $>0.05$ (Table 2). The mean gingival index at base line in controls and test group was $2.36 \pm 0.39$ and $2.28 \pm 0.40$, p-value > 0.477 while mean gingival index at $2 \mathrm{nd}$ week in control and test group was $.62 \pm 0.18$ and $0.46 \pm 0.20$ with no significant difference, p-value $<0.006$ (Table 3). Mean percentage change in gingival index in control and test group were $72.95 \pm 9.67 \%$ and $79.54 \pm 8.68 \%$, with higher percentage change in test group p-value $<0.015$ (Fig. 1). The mean plaque index at base line in controls and test group was $2.67 \pm 0.23$ and $2.70 \pm 0.25$, p-value $>0.624$ while mean plaque index at 2 nd week in control and test group was $.62 \pm 0.17$ and $.59 \pm 0.17$ with no significant difference, p-value $<0.053$ (Table 3). Mean percentage change in plaque index in control and test group were 76.41 $\pm 7.28 \%$ and $78.04 \pm 6.89 \%$, with higher percentage change in test group p-value $<0.422$ (Fig. 2). In this study statistically no significant difference between both groups, however, there is marked reduction percentage wise in the Gingival and Plaque Index score (Fig. 1-2). Regarding side effects, clinically no significant local side effects were noticed in both groups.

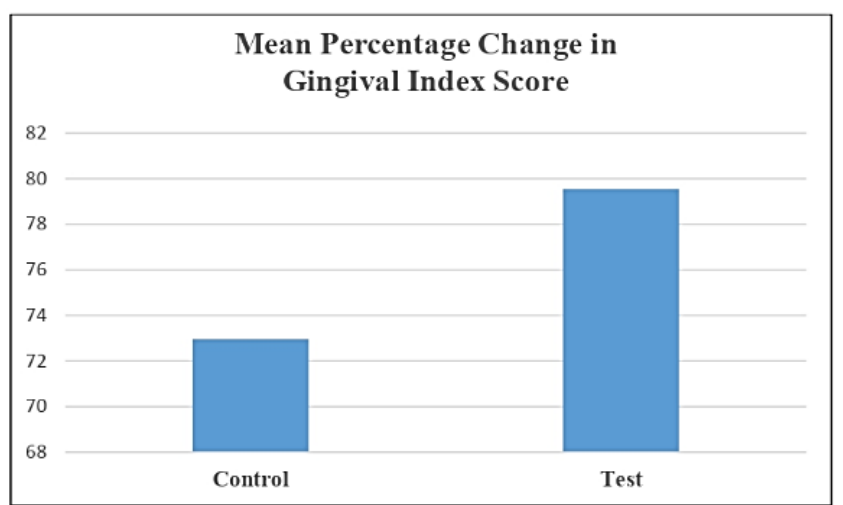

Fig. 1: Comparison mean percentage change in Gingival index in both groups

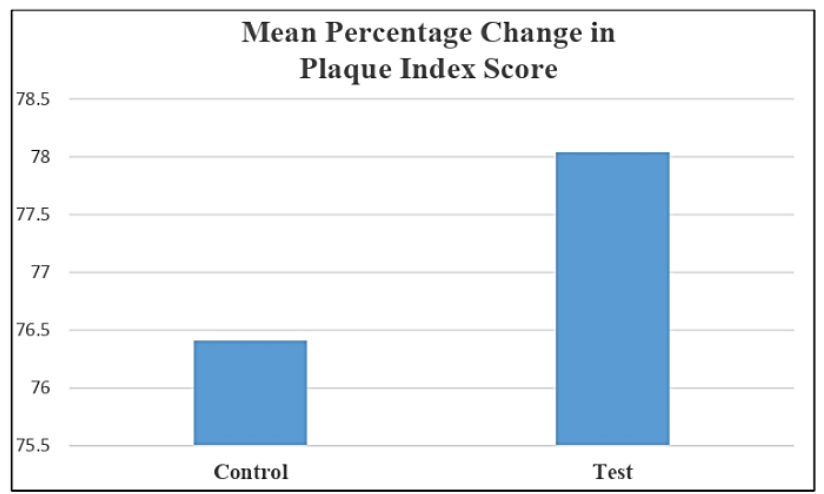

Fig. 2: Comparison mean percentage change in plaque index in both groups

\section{Discussion}

Many adverse effects were observed during the use of mouthwashes, as staining is one of the complication specially use of Chlorhexidine Mouthwash (MW). The staining increase in tea, coffee, wine, and cigarettes users when using Chlorhexidine mouthwashes (Van MaanenSchakel, Slot, Bakker, \& Van der Weijden, 2012). In users of Chlorhexidine mouthwashes also noted the side effect of bitter taste (Frank, Gent, \& Hettinger, 2001).

Van Strydonck (2012) in a systemic review concluded that long term use of "Chlorhexidine Mouthwashes" are contraindicated because of change in taste, formation of calculus and extrinsic tooth stinging as daily oral home care measures. (Van Strydonck, Slot, Van der Velden, \& Van der Weijden, 2012).

Gunsolley (2006) in a meta-analysis found that CHX mouthwash used extensively and evaluated that decrease in Gingival Inflammation at 6 months was $28.7 \%$ (Gunsolley, 2006), while James et al. (2017) established that use of CHX mouthwash for 04 weeks or more leading to the tooth staining. In addition to some other side effects like calculus formation change in taste and effects on the oral mucosa were observed in the studies include in the review (James et al., 2017).

Strydonck et al. (2012) did a systematic review found that teeth staining was the record more in 19 , calculus formation in 06 and change of taste feeling 07 articles out of 30. (Van Strydonck et al., 2012) (Van Maanen-Schakel et al., 2012). Adverse effects of prescribing mouthwash should be considered for the patient's safety point of view. Therefore, studies on the use of Biorepair mouthwash found to be safe considering the all these side effects of CHX. (Takenaka, Ohsumi, \& Noiri, 2018)

Saverio Cosola (2010) did a study on mouthwash having "nanohydroxyapatite (Zn-nHAp) and zinc Lpyrrolidone carboxylate (Zn-PCA)" compare with "Chlorhexidine" (CHX), effect of microbial presence on surgical sutures. It was found in this study that similar antibacterial effectiveness to the mouthwash containing chlorhexidine but without consequences of CHX (Cosola et al., 2017). The same observation was concluded in our study, that Zinc-carbonate hydroxyapatite nanocrystals $(\mathrm{ZCH})$ mouthwash is safe to use comparing with "Chlorhexidine"

Hannig, C (2013), did a study to evaluate the effect of mouthwash containing hydroxyapatite microclusters in reducing the bacterial establishment on enamel surfaces (C. Hannig et al., 2013).

Claudio Palmieri (2013) did a study to examine the effect of zinc-carbonate hydroxyapatite ( $\mathrm{Zn}-\mathrm{CHA})$ on dental biofilm formation, found $\mathrm{Zn}-\mathrm{CHA}$ is effective against $\mathrm{S}$. mutans biofilm (Palmieri, Magi, Orsini, Putignano, \& Facinelli, 2013). 
Table 1: Descriptive statistics of age (years) in both groups

\begin{tabular}{|c|c|c|c|c|}
\hline & Study groups & Mean & S.D & p-value \\
\hline \multirow{2}{*}{$\begin{array}{c}\text { Age groups } \\
\text { (years) }\end{array}$} & Control $(\mathbf{n = 2 5})$ & 41.04 & 6.91 & \multirow{2}{*}{0.396} \\
\cline { 2 - 4 } & Test $(\mathbf{n = 2 5})$ & 39.52 & 5.58 & \\
\hline
\end{tabular}

Table 2: Demographic characteristics in both groups

\begin{tabular}{|l|c|c|c|c|}
\hline Groups & Characteristics & Frequency & Percent & p-value \\
\hline \multirow{3}{*}{ Control } & Male & 16 & $64 \%$ & \\
\cline { 2 - 4 } Test & Female & 9 & $36 \%$ & \multirow{2}{*}{0.05} \\
\cline { 2 - 4 } & Male & 17 & $68 \%$ & \\
\hline
\end{tabular}

Table 3: Comparison of Gingival index and plaque index at different follow-ups in both groups

\begin{tabular}{|c|c|c|c|c|}
\hline & Study groups & Mean & S.D & p-value \\
\hline \multirow{2}{*}{$\begin{array}{c}\text { Gingival index at } \\
\text { base line }\end{array}$} & Control $(n=25)$ & 2.36 & 0.39 & \multirow{2}{*}{0.482} \\
\hline & Test $(n=25)$ & 2.28 & 0.40 & \\
\hline \multirow{2}{*}{$\begin{array}{c}\text { Gingival index at } \\
\text { week } 2\end{array}$} & Control $(n=25)$ & 0.62 & 0.18 & \multirow{2}{*}{0.001} \\
\hline & Test $(n=25)$ & 0.46 & 0.20 & \\
\hline \multirow{2}{*}{$\begin{array}{l}\text { Percentage change in } \\
\text { Gingival index }\end{array}$} & Control $(n=25)$ & 72.95 & 9.67 & \multirow{2}{*}{0.001} \\
\hline & Test $(n=25)$ & 79.54 & 8.68 & \\
\hline \multirow{2}{*}{$\begin{array}{l}\text { Plaque index at } \\
\text { base line }\end{array}$} & Control $(n=25)$ & 2.67 & 0.23 & \multirow{2}{*}{0.624} \\
\hline & Test $(n=25)$ & 2.70 & 0.25 & \\
\hline \multirow{2}{*}{$\begin{array}{l}\text { Plaque index at } \\
\text { week } 2\end{array}$} & Control $(n=25)$ & 0.62 & 0.14 & \multirow{2}{*}{0.001} \\
\hline & Test $(n=25)$ & 0.58 & 0.17 & \\
\hline \multirow{2}{*}{$\begin{array}{l}\text { Percentage change in } \\
\text { Plaque index }\end{array}$} & Control $(n=25)$ & 76.41 & 7.28 & \multirow{2}{*}{0.001} \\
\hline & Test $(n=25)$ & 78.04 & 6.89 & \\
\hline
\end{tabular}

Salwa A. Hegazy (2016) did study to discover that Biorepair mouthwash decrease the plaque formation from baseline to the use of the 1 st week and found significant after six weeks. Also Salwa revealed that gingival inflammation scores significant decrease from the baseline to fourth and sixth weeks along with remineralizing effect. (Salwa A. Hegazy (2016).). Results of the Salwa A. Hegazy study recommend that Biorepair mouthwash can be used as an substitute for both fluoride and chlorhexidine mouthwashes (Hegazy \& Salama, 2016).

After the use of certain mouthwashes, effects of roughness on the surfaces of dental appliances (implants/orthodontic brackets) in the oral cavity was observed. On the other hand, in a vitro study done by Lelli M (2013) was found that a mouthwash containing zincsubstituted carbonate-hydroxyapatite decrease the coarsest on the surfaces and prevent microbial growth on the "Implant-Supported" appliances. (Lelli et al., 2013)

Consuelo Sanavia et al. (2017) concluded mouthwash containing zinc-substituted CHA prevents the corrosion of the dental implants and bacterial accumulation on the surfaces (Sanavia et al., 2017).

The main limitation of our study is the short follow-up time ( 2 weeks), However, the long term study can be our next step to evaluate the safety of Zinc-carbonate hydroxyapatite nanocrystals $(\mathrm{ZCH})$ mouthwash for long term use ( $>4$ weeks).

\section{Conclusion}

In our study there is no strong significant difference found in control and test mouthwashes. Considering the side effects of chlorhexidine (CHX) and in the light of other researches, our study strongly advocates to efficacy of Biorepair mouthwash were effective in controlling Dental Plaque and Gingivitis when compared chlorhexidine (CHX). The finding of this study proposed that Biorepair mouthwash safely used as a substitute for chlorhexidine. This Biorepair mouthwash can be used as a multipurpose action mouthwash, because it has properties of remineralizing effects in caries prevention.

\section{Acknowledgments}

The authors want to acknowledge Dr. Asif Hanif (PhD) Associate Professor of Biostatistics, University institute of Public health, Faculty of Allied health Science. The University of Lahore, Pakistan for helping in statistical analysis. The authors also want to acknowledge Mr. Bashir Shakib Al Jabri, Dr. Ezzat Hamid Mabrouk and Mr. Abdul Rahman Abdul Dam of Medical \& Pharmaceutical Services, KSA for providing samples in this research study. 


\section{Authors' contributions}

Both authors in this study participated in planning the study, clinical examination and data collection and writing and reviewing the article.

\section{Conflicts of interest}

The authors have stated explicitly that there are no conflicts of interest in connection with this article.

\section{Statement of sources of funding for the study}

The authors received no specific funding for this work.

\section{References}

1. Brading M., \& Marsh P. The oral environment: the challenge for antimicrobials in oral care products. Int Dent $J$ 2003;53(S6P1):353-62.

2. Cosola S., Marconcini S., Giammarinaro E., Marchisio O., M., L., Roveri N., \& Genovesi A. M. Antimicrobial efficacy of mouthwashes containing zinc-substituted nanohydroxyapatite and zinc L-pyrrolidone carboxylate on suture threads after surgical procedures. J Oral Sci Rehabil 2017;3(4):24-30.

3. Cummins D. (1997). Vehicles: how to deliver the goods. Periodontol 2000;15(1):84-99.

4. Cummins D., \& Creeth J. Delivery of antiplaque agents from dentifrices, gels, and mouthwashes. J Dent Res 1992;71(7):1439-49.

5. Frank M. E., Gent J. F., \& Hettinger T. P. Effects of chlorhexidine on human taste perception. Physiol Behav 2001;74(1-2);85-99.

6. Gunsolley J. C. A meta-analysis of six-month studies of antiplaque and antigingivitis agents. J Am Dent Assoc 2006;137(12): 164957.

7. Hannig C., Basche, S., Burghardt, T., Al-Ahmad, A., \& Hannig, M. (2013). Influence of a mouthwash containing hydroxyapatite microclusters on bacterial adherence in situ. Clin Oral Investigations 2013;17(3):805-14.

8. Hannig M., \& Hannig C. Nanomaterials in preventive dentistry. Nat Nanotechnol 2010;5(8):565.

9. Hassanein O. E., \& El-Brolossy T. An investigation about the remineralization potential of bio-active glass on artificially carious enamel and dentin using Raman spectroscopy. Egypt $J$ Solids 2006;29(1):69-80.

10. Hegazy S. A., \& Salama R. I. Antiplaque and remineralizing effects of Biorepair mouthwash: A comparative clinical trial. Pediatric Dent J, 2016;26(3):89-94.

11. Jackson R. J. Metal salts, essential oils and phenols-old or new? Periodontol 2000;15(1):63-73.

12. James P, Worthington H. V, Parnell C, Harding M, Lamont T, Cheung A, Riley P. Chlorhexidine mouthrinse as an adjunctive treatment for gingival health. Cochrane Database Syst Rev 2017(3). doi: 10.1002/14651858.CD008676.pub2.

13. Kuboniwa M, \& Lamont R. J. Subgingival biofilm formation. Periodontol 2000;52(1):38-52.

14. Lelli M, Marchisio O, Foltran I, Genovesi A, Montebugnoli G, Marcaccio M, Roveri, N. (2013). Different corrosive effects on hydroxyapatite nanocrystals and amine fluoride-based mouthwashes on dental titanium brackets: a comparative in vitro study. Int J Nanomedicine 2013;8:307.

15. Loe H., \& Silness J. Periodontal Disease in Pregnancy. I. Prevalence and Severity. Acta Odontol Scand 1963;21:533-51.

16. Löe H., Theilade E., \& Jensen S. B. (1965). Experimental gingivitis in man. J Periodontol 1965;36(3):177-87.

17. Marsh P. Dental plaque as a microbial biofilm. Caries Res, 2004;38(3):204-11.

18. Marsh P. Controlling the oral biofilm with antimicrobials. $J$ Dent 2010;38:S11-S5.
19. Marsh P. Contemporary perspective on plaque control. $\mathrm{Br}$ Dent J 2012;212(12):601.

20. Moran J. M. Chemical plaque control-prevention for the masses. Periodontol 2000;15(1):109-17.

21. Noiri Y, Li L., \& Ebisu S. The localization of periodontaldisease-associated bacteria in human periodontal pockets. $J$ Dent Res, 2001;80(10):1930-4.

22. Page R. C., Offenbacher S., Schroeder H. E., Seymour G. J., \& Kornman K. S. Advances in the pathogenesis of periodontitis: summary of developments, clinical implications and future directions. Periodontol 2000;14(1):216-48.

23. Palmieri, C., Magi, G., Orsini, G., Putignano, A., \& Facinelli, B. (2013). Antibiofilm activity of zinc-carbonate hydroxyapatite nanocrystals against Streptococcus mutans and mitis group Streptococci. Curr Microbiol 2013;67(6):679-81.

24. Pitts N. B, Zero D. T, Marsh P. D, Ekstrand K, Weintraub J. A, Ramos-Gomez F et al. Dental caries. Natu Rev Dis Primers 2017;3:17030

25. Quigley G. A, \& Hein J. W. Comparative cleansing efficiency of manual and power brushing. J Am Dent Assoc 1962;65:26-9.

26. Roveri N., Battistella E., Foltran I., Foresti E., Iafisco M., Lelli M. et al. Synthetic biomimetic carbonate-hydroxyapatite nanocrystals for enamel remineralization. Adv Materials Res 2008;47-50:821-4.

27. Saad S., Greenman J., \& Shaw H. Comparative effects of various commercially available mouthrinse formulations on oral malodour. Oral Dis 2011;17(2):180-6.

28. Sanavia C., Tatullo M., Bassignani J., Cotellessa S., Fantozzi G, Acito G et al. Remineralization Strategies in Oral Hygiene: A Position Paper of Italian Society of Oral Hygiene SciencesSISIO Working Group. Open Dent J 2017;11:527.

29. Seymour G., Ford P, Cullinan M., Leishman S, \& Yamazaki K. Relationship between periodontal infections and systemic disease. Clin Microbiol Infect, 2007;13:3-10.

30. Socransky S. S., \& Haffajee A. D. Dental biofilms: difficult therapeutic targets. Periodontol 2000;28(1):12-55.

31. Sreenivasan, P., \& Gaffar, A. Antiplaque biocides and bacterial resistance: a review. J Clin Periodontol 2002;29(11):965-74.

32. Takahashi N., \& Nyvad B. The role of bacteria in the caries process: ecological perspectives. J Dent Res, 2011;90(3):294303.

33. Takenaka S., Ohsumi T., \& Noiri Y. Evidence-based strategy for dental biofilms: Current evidence of mouthwashes on dental biofilm and gingivitis Jpn Dent Sci Rev 2018;55(1):3340. doi: 10.1016/j.jdsr.2018.07.001

34. Talbott K., Mandel I., \& Chilton N. Reduction of baseline gingivitis scores with repeated prophylaxes. The J Prev Dent 1977;4(6):28-9.

35. Turesky S., Gilmore N. D., \& Glickman I. Reduced plaque formation by the chloromethyl analogue of victamine C. $J$ Periodontol, 1970;41(1):41-3.

36. Van Maanen-Schakel, N., Slot D., Bakker E., \& Van der Weijden $\mathrm{G}$. The effect of an oxygenating agent on chlorhexidine-induced extrinsic tooth staining: a systematic review. Int J Dent Hyg 2012;10(3):198-208.

37. Van Strydonck D. A., Slot D. E., Van der Velden U., \& Van der Weijden F. Effect of a chlorhexidine mouthrinse on plaque, gingival inflammation and staining in gingivitis patients: a systematic review. J Clin Periodontol, 2012;39(11):1042-55.

How to cite this article: Asmari DA, Almutairi A.
Clinical evaluation of zinc-carbonate hydroxyapatite
nanocrystals mouthwash in controlling plaque induced
gingivitis: A randomized clinical trial. Int J Periodontol
Implantol 2019;4(3):98-102.

